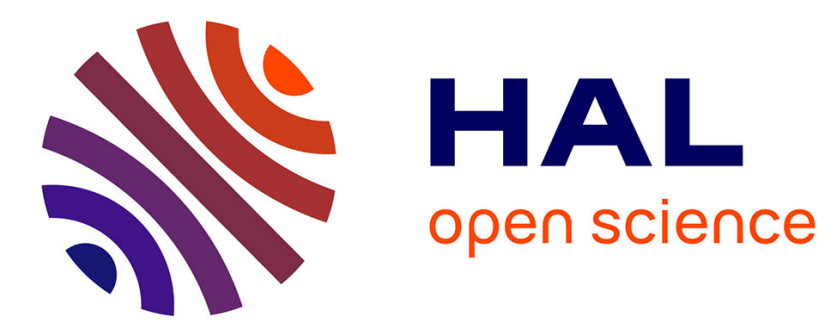

\title{
CONSTRAINED MANIPULABILITY FOR HUMANOID ROBOTS USING VELOCITY POLYTOPES
}

\author{
Philip Long, Taşkin Padir
}

\section{- To cite this version:}

Philip Long, Taşkin Padir. CONSTRAINED MANIPULABILITY FOR HUMANOID ROBOTS USING VELOCITY POLYTOPES. International Journal of Humanoid Robotics, 2020. hal-03189852

\section{HAL Id: hal-03189852 https://hal.science/hal-03189852}

Submitted on 5 Apr 2021

HAL is a multi-disciplinary open access archive for the deposit and dissemination of scientific research documents, whether they are published or not. The documents may come from teaching and research institutions in France or abroad, or from public or private research centers.
L'archive ouverte pluridisciplinaire HAL, est destinée au dépôt et à la diffusion de documents scientifiques de niveau recherche, publiés ou non, émanant des établissements d'enseignement et de recherche français ou étrangers, des laboratoires publics ou privés. 


November $16, \quad 2019 \quad 14: 43 \quad$ WSPC/INSTRUCTION $\quad$ FILE

\title{
CONSTRAINED MANIPULABILITY FOR HUMANOID ROBOTS USING VELOCITY POLYTOPES
}

\author{
Philip Long \\ Department of Electrical and Computer Engineering, \\ Northeastern University, Boston, Massachusetts 02115, USA. \\ p.long@northeastern.edu \\ Taşkın Padır \\ Department of Electrical and Computer Engineering, \\ Northeastern University, Boston, Massachusetts 02115, USA. \\ t.padir@northeastern.edu
}

\begin{abstract}
Robot performance measures are important tools for quantifying the ability to carry out manipulation tasks. Generally, these measures examine the system's kinematic transformations from configuration to task space. This means that environmental constraints are neglected in spite of the significant effects they may have on the robot's admissible motions. In this paper, we propose a new measure called the constrained manipulability polytope (CMP) that considers the system's kinematic structure, including closed chains or composite sub-mechanisms, joint limits and the presence of obstacles. For an illustrative planar case, we demonstrate how the CMP can evaluate a robot's performance in a cluttered scene and how this evaluation can be extrapolated to obtain a workspace visualization. Additionally we show the advantages and limitations of the CMP compared to the state of the art. Furthermore, the method is demonstrated both in simulation and experimentally for NASA's Valkyrie robot. We show how the CMP provides a measure for single-arm and dual-arm manipulation tasks, analyze the workspace and be used to optimize the robot's posture.
\end{abstract}

Keywords: Manipulability Polytopes; Constrained Workspace Analysis; Humanoid Robots; Posture Analysis.

\section{Introduction}

A robot performance index is a metric that can be used for synthesis, base placement and task planning applications ${ }^{1,2,3}$. These measures can be classified as local, for instance manipulability ${ }^{4}$ or global such as workspace analysis ${ }^{5}$. Local indexes are workspace independent and can be used to select a configuration based on a robot's inherent capability. In contrast, if the workspace is well known a robot's overall performance can be analyzed, for example by discretization reachability can be verified for each voxel ${ }^{5}$. Thus global indexes can be used to compare manipulator suitability and/or posture for a particular task. While clearly connected, ${ }^{6}$ has shown that a good local index does not necessarily imply a good global performance.

It is desirable to use local indexes since they provide a more generic solution 
and hence may be exploited in control frameworks without prior workspace knowledge. Additionally, many global measures are based on the evaluation/integration of a local index across a workspace. For these reasons, much research has focused on extending local measures such as the manipulability ellipsoid ${ }^{4}$ to include various constraints. Joint position limits can severely limit a robot's manipulation capabilities $^{7}$ and thus should be considered in developing evaluation metrics. $\mathrm{In}^{8}$, the author proposes to modify the manipulability index by using a penalty function based on proximity to positional joint limits. Likewise, joint limits are taken into account in $^{3}$ in this case by using an augmented Jacobian matrix. Since the classical manipulability measure considers a unit hyper-sphere of joint velocities, individual velocity limits have been taken into account in $^{9}$ by scaling the Jacobian matrix. Other notable extensions have included the robot's dynamic constraints ${ }^{10,11}$. For humanoid robots, improvements on local measures can be obtained by including the effects of contact while evaluating the dynamic manipulability of a humanoid's center of mass ${ }^{12,13}$. Moreover $i^{13}$, a scaling matrix is proposed to enforce either joint torque and/or acceleration limits.

The work most related to our approach is presented in ${ }^{5,14,15}$, where the manipulability ellipsoid is extended to include additional environmental constraints. The authors' method considers joint position limits and the detrimental effects of nearby obstacles. To include these constraints in the manipulability ellipsoid, the Jacobian is first altered by a scaling matrix. Secondly, a set of augmented Jacobian matrices is computed that considers workspace movements in all possible Cartesian directions. The authors propose a range of different applications, such as fast inverse kinematic solutions and grasp evaluations, for this index by demonstrating how it can be extended across a workspace to generate a manipulability map. Instead of the hyperoctants approach, manipulability polytopes provide a more natural and intuitive way of representing velocity limits in the Cartesian space, as first proposed in ${ }^{16}$. Indeed, polytopes give an exact representation of the robot's velocity bounds instead of the ellipsoids' approximation ${ }^{17,18}$. Moreover, for the redundant case, polytopes are less susceptible to errors. For instance, ${ }^{19}$ proves that an ellipsoid does not represent the isotropic capabilities of a planar 2-DOF robot. In fact ${ }^{20}$ shows that using the ellipsoid to analyze Cartesian-space positioning errors based on corresponding joint errors, leads to unrealistic results. Additionally, polytopes have the advantage of being standard geometric objects and thus are much easier to transform and combine as demonstrated in Section 2.3. In fact, there are several examples where diverse constraints, defined by a set of inequality or equality equations, have been incorporated into polytopes. For instance, mobile robot toppling constraints have been integrated into the available wrench set for a cable-driven parallel robot $i^{21,22}$. Alternatively friction constraints can be added after linearization ${ }^{23}$. Polytopes have been neglected as a performance index typically due to the perceived computational $\operatorname{cost}^{14}$, which nowadays presents less of a limitation.

In this paper, we extend our approach ${ }^{24}$ to measure a humanoid robot's performance based on manipulability polytopes. First, a comparison is carried out with a 
state-of-the-art algorithm that evaluates reduced manipulability. We establish how our measure can be used to analyze a humanoid robot's workspace and evaluate single-arm and dual-arm manipulation operations. In contrast to previous techniques, both task- and joint-space constraints on each link are considered. While the manipulability for a humanoid robot typically treats the arms as separate serial manipulators ${ }^{25}$, we demonstrate how polytopes can be combined to consider the resulting manipulability of serial and parallel chains. Finally, we show how the resulting metric can be incorporated into a constrained optimization in order to find optimal postures. This paper is organized as follows. In Section 2, the manipulability concept is recalled and the proposed method to fuse manipulability polytopes is shown. In Section 3, our approach for embedding the task constraints into the polytope is detailed and compared with the state of the art. Section 4 presents the case study of a humanoid robot operating in a highly-constrained environment. Finally, in Section 5, the conclusions are drawn and future work is outlined.

\section{Manipulability}

\subsection{Manipulability Ellipsoid}

Consider an $n$ degree-of-freedom (DOF) manipulator operating in $m$ dimensional space. Let $\boldsymbol{\nu}_{n}$ denote the twist at the end effector, comprising 3 translational and 3 angular velocities defined respectively as $\mathbf{v}$ and $\boldsymbol{\omega} . \boldsymbol{\nu}_{n}$ is obtained as

$$
\boldsymbol{\nu}_{n}=\left[\begin{array}{c}
\mathbf{v} \\
\boldsymbol{\omega}
\end{array}\right]=\mathbf{J}_{n} \dot{\mathbf{q}}
$$

where $\mathbf{J}_{n} \in \mathbb{R}^{6 \times n}$ is the Jacobian matrix and $\dot{\mathbf{q}}=\left[\dot{q}_{1}, \dot{q}_{2} \ldots \dot{q}_{n}\right]^{T}$ is the joint velocity vector. In this paper, all quantities unless otherwise stated are represented in the fixed world frame. The manipulability ellipsoid ${ }^{4}$, denoted as $\mathcal{E}$, measures the robot's capacity to transmit velocities and forces from joint space to task space and can be obtained by considering the joint velocities in the unit hypersphere i.e.,

$$
\dot{\mathbf{q}}^{T} \dot{\mathbf{q}} \leq 1
$$

By substituting Eq. (1) into Eq. (2), the task-space ellipsoid is described by

$$
\mathcal{E}=\left\{\boldsymbol{\nu}_{n}^{T}\left(\mathbf{J}_{n} \mathbf{J}_{n}^{T}\right)^{-1} \boldsymbol{\nu}_{n} \leq 1\right\}
$$

The eigenvectors of $\mathbf{J}_{n} \mathbf{J}_{n}^{T}$ define the principal axes of $\mathcal{E}$ while their magnitude is defined by the corresponding singular values of $\mathbf{J}_{n}$. By studying the volume of $\mathcal{E}$, a measure of the manipulator's performance is obtained, albeit with several known limitations such as dimensional dependency ${ }^{26}$. 


\subsection{Manipulability Polytope}

A polytope, $\mathcal{P}$ can be represented as the convex hull of its vertex set $(\mathcal{V}$ representation), i.e.,

$$
\mathcal{P}^{V}=\left\{\mathbf{x}: \mathbf{x}=\sum_{i=1}^{n} \alpha_{i} \mathbf{y}_{i} \mid \alpha_{i} \geq 0, \sum_{i=1}^{n} \alpha_{i}=1\right\}
$$

where $\mathbf{y}_{i}$ denotes the $i^{\text {th }}$ element of the vertex set and $\mathbf{x}$ is any point inside $\mathcal{P}$. Equivalently, $\mathcal{P}$ can be defined as the volume bounded by a finite number of halfspaces ( $\mathcal{H}$-representation)

$$
\mathcal{P}^{H}=\mathbf{A x} \leq \mathbf{b},
$$

where A contains the half-spaces' normals and $\mathbf{b}$ is the shifted distance from the origin along the normal. Converting from $\mathcal{V}$ and $\mathcal{H}$ representations can be carried out in several ways, for example using the double description ${ }^{\mathrm{a}}$ method $^{28}$.

The polytope representing joint velocities for an $n$-DOF robot, denoted by $\mathcal{Q}$, is written in $\mathcal{H}$-representation as

$$
\mathcal{Q}^{H}=\left[\begin{array}{c}
\mathbf{I}_{n} \\
-\mathbf{I}_{n}
\end{array}\right] \dot{\mathbf{q}} \leq\left[\begin{array}{c}
\dot{\mathbf{q}}_{\text {max }} \\
-\dot{\mathbf{q}}_{\text {min }}
\end{array}\right],
$$

where $\mathbf{I}_{n}$ is the $n \times n$ identity matrix and $\dot{\mathbf{q}}_{\text {max }}$ and $\dot{\mathbf{q}}_{\text {min }}$ denote the robot's maximum and minimum joint velocities respectively. The equivalent polytope defined by its vertices is written as

$$
\mathcal{Q}^{V}=\left\{\dot{\mathbf{q}}_{1}^{v}, \dot{\mathbf{q}}_{2}^{v}, \ldots, \dot{\mathbf{q}}_{2^{n}}^{v}\right\}
$$

where $\dot{\mathbf{q}}_{i}^{v}$ denotes the $i^{\text {th }}$ vertex of $\mathcal{Q}$. The vertices can be arranged in matrix form as

$$
\mathbf{Q}=\left[\begin{array}{c}
\dot{\mathbf{q}}_{1}^{v} \\
\dot{\mathbf{q}}_{2}^{v} \\
\vdots \\
\dot{\mathbf{q}}_{2^{n}}^{v}
\end{array}\right]=\left[\begin{array}{ccccc}
\dot{q}_{1}^{\min } & \dot{q}_{2}^{\min } & \ldots & \dot{q}_{n-1}^{\min } & \dot{q}_{n}^{\min } \\
\dot{q}_{1}^{\text {min }} & \dot{q}_{2}^{\text {min }} & \ldots & \dot{q}_{n-1}^{\text {min }} & \dot{q}_{n}^{\text {max }} \\
\vdots & \vdots & \vdots \\
\dot{q}_{1}^{\max } & \dot{q}_{2}^{\max } & \ldots & \dot{q}_{n-1}^{\max } & \dot{q}_{n}^{\max }
\end{array}\right]
$$

The convexity of a polytope is preserved under affine transformation, i.e., a linear transformation applied to $\mathbf{Q}$ is a convex combination of the same linear transformation applied to the vertices. Thus, a manipulability polytope (MP), denoted as $\mathcal{P}$, representing the Cartesian-space velocities can be obtained by combining Eq. (1) and Eq. (8). $\mathcal{P}$ 's vertex set representation is given as

$$
\mathcal{P}^{V}=\left\{\boldsymbol{\nu}_{1}^{v} \ldots \boldsymbol{\nu}_{2^{n}}^{v}\right\}=\left\{\mathbf{J}_{n} \dot{\mathbf{q}}_{1}^{v} \ldots \mathbf{J}_{n} \dot{\mathbf{q}}_{2^{n}}^{v}\right\}
$$

and its volume, denoted as $w_{p}$, can be used as an indicator of robot performance.

\footnotetext{
${ }^{a}$ In this work, for humanoid robot analysis, we use the C++ wrapper for Fukuda's cdd library available at https://github.com/vsamy/eigen-cdd, while the MATLAB(C) computations (planar comparison case) used the Multi-Parametric Toolbox 3.0. ${ }^{27}$
} 


\subsection{Manipulability of Complex Mechanisms}

In the following, we show how the MP of several mechanisms can be combined to evaluate the performance of a composite mechanism. In brief:

- $\mathcal{P}=\mathcal{P}_{1} \oplus \mathcal{P}_{2} \ldots \oplus \mathcal{P}_{n}$, namely, the polytope of a mechanism composed of $n$ submechanisms in series is the Minkowski sum, denoted by $\oplus$, of each sub-mechanism's polytope.

- $\mathcal{P}=\mathcal{P}_{1} \cap \mathcal{P}_{2} \ldots \cap \mathcal{P}_{n}$, namely, the polytope of a mechanism composed of $n$ sub-mechanisms in parallel is the intersection of each sub-mechanism's polytope. This relation is first proposed in ${ }^{16}$ for cooperative robots manipulating a common object.

\subsubsection{Manipulability Compositions for Serial Mechanisms}

Without loss of generality, suppose the $n$-DOF mechanism, described by Eq. (1), is composed a serial arm mounted on a mobile base. The joint positions $\mathbf{q} \in \mathbb{R}^{n \times 1}$ and can be separated into $k$ variables belonging to the serial arm and $n-k$ components belonging to the mobile base, denoted respectively as $\mathbf{q}^{a r m}$ and $\mathbf{q}^{m b}$. The corresponding velocity variables are given as $\dot{\mathbf{q}}, \dot{\mathbf{q}}^{\text {arm }}$ and $\dot{\mathbf{q}}^{m b}$. Thus, Eq. (1) becomes

$$
\boldsymbol{\nu}=\mathbf{J} \dot{\mathbf{q}}=\left[\begin{array}{ll}
\mathbf{J}_{a r m} & \mathbf{J}_{m b}
\end{array}\right]\left[\begin{array}{c}
\dot{\mathbf{q}}^{a r m} \\
\dot{\mathbf{q}}^{m b}
\end{array}\right] \text {. }
$$

$\mathbf{J}_{\text {arm }} \in \mathbb{R}^{m \times k}$ and $\mathbf{J}_{m b} \in \mathbb{R}^{m \times(n-k)}$ are the Jacobian matrices of the submechanisms. From Eq. (6) and Eq. (8), the joint-space polytope's vertex sets are given as

$$
\mathbf{Q}_{a r m}=\left[\dot{\mathbf{q}}_{1}^{a r m} \dot{\mathbf{q}}_{2}^{a r m} \ldots \dot{\mathbf{q}}_{2^{k}}^{a r m}\right]^{T} \quad \mathbf{Q}_{m b}=\left[\dot{\mathbf{q}}_{1}^{m b} \dot{\mathbf{q}}_{2}^{m b} \ldots \dot{\mathbf{q}}_{2^{n-k}}^{m b}\right]^{T},
$$

where $\dot{\mathbf{q}}_{i}^{a r m}$ and $\dot{\mathbf{q}}_{j}^{m b}$ denote the $i^{\text {th }}$ and $j^{\text {th }}$ vertices in $\mathcal{Q}_{\text {arm }}$ and $\mathcal{Q}_{m b}$ respectively. From Eq. (9), the corresponding task space vertex sets are given as

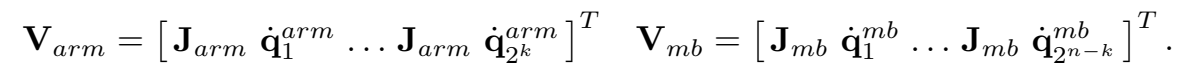

$\mathbf{V}_{\text {arm }} \in \mathbb{R}^{2^{k} \times m}$ and $\mathbf{V}_{m b} \in \mathbb{R}^{2^{n-k} \times m}$ define the convex MPs, $\mathcal{P}_{\text {arm }}$ and $\mathcal{P}_{m b}$ of the serial arm and the mobile base respectively, i.e.,

$$
\begin{aligned}
\mathcal{P}_{a r m}^{V} & =\left\{\mathbf{J}_{a r m} \dot{\mathbf{q}}_{1}^{a r m}, \mathbf{J}_{a r m} \dot{\mathbf{q}}_{2}^{a r m}, \ldots \mathbf{J}_{a r m} \dot{\mathbf{q}}_{2^{k}}^{a r m}\right\}, \\
\mathcal{P}_{m b}^{V} & =\left\{\mathbf{J}_{m b} \dot{\mathbf{q}}_{1}^{m b}, \mathbf{J}_{m b} \dot{\mathbf{q}}_{2}^{m b}, \ldots \mathbf{J}_{m b} \dot{\mathbf{q}}_{2^{m-k}}^{m b}\right\} .
\end{aligned}
$$

The Minkowski sum of $\mathcal{P}_{1}$ and $\mathcal{P}_{2}$ is formed by adding each vector in $\mathcal{P}_{1}$ to each vector in $\mathcal{P}_{2}$, i.e., $\mathcal{P}_{1} \oplus \mathcal{P}_{2}=\left\{\mathbf{a}+\mathbf{b} \mid \mathbf{a} \in \mathcal{P}_{1}, \mathbf{b} \in \mathcal{P}_{2}\right\}$. Since MP's are by definition convex the Minkowski sum can be computed by taking the pairwise summation of 
the vertices. The resulting set is of dimension $\left(2^{k} \cdot 2^{n-k} \times m\right)$, i.e., $2^{n} \times m$

$$
\begin{aligned}
& \mathcal{P}_{a r m}^{V} \oplus \mathcal{P}_{m b}^{V}=\left\{\mathbf{J}_{a r m} \dot{\mathbf{q}}_{1}^{a r m}+\mathbf{J}_{m b} \dot{\mathbf{q}}_{1}^{m b}, \mathbf{J}_{a r m} \dot{\mathbf{q}}_{1}^{a r m}+\mathbf{J}_{m b} \dot{\mathbf{q}}_{2}^{m b}, \ldots\right. \\
& \mathbf{J}_{a r m} \dot{\mathbf{q}}_{1}^{a r m}+\mathbf{J}_{m b} \dot{\mathbf{q}}_{2^{n-k}}^{m b}, \\
& \vdots \\
& \mathbf{J}_{a r m} \dot{\mathbf{q}}_{2^{k}}^{a r m}+\mathbf{J}_{m b} \dot{\mathbf{q}}_{1}^{m b}, \mathbf{J}_{a r m} \dot{\mathbf{q}}_{2^{k}}^{a r m}+\mathbf{J}_{m b} \dot{\mathbf{q}}_{2}^{m b}, \ldots \\
&\left.\mathbf{J}_{a r m} \dot{\mathbf{q}}_{2^{k}}^{a r m}+\mathbf{J}_{m b} \dot{\mathbf{q}}_{2^{n-k}}^{m b}\right\}
\end{aligned}
$$

As seen in Eq. (8) a vertex of the $n$-dimensional polytope $\mathcal{Q}$ is a vector formed by the vertices of its sub-mechanisms, and supposing judicious labeling of vertices, the following expression can always be obtained

$$
\mathbf{q}_{i}^{v}=\left[\mathbf{q}_{i}^{a r m} \mathbf{q}_{i}^{m b}\right] \quad \forall i=1 \ldots n
$$

Substituting Eq. (16) and Eq. (10) into Eq. (15), it can be shown that

$$
\mathcal{P}_{\text {arm }}^{V} \oplus \mathcal{P}_{m b}^{V}=\left\{\mathbf{J} \dot{\mathbf{q}}_{1}^{v}, \mathbf{J} \dot{\mathbf{q}}_{2}^{v}, \ldots, \mathbf{J} \dot{\mathbf{q}}_{n}^{v}\right\}=\mathcal{P}^{V} .
$$

demonstrating that the MP of a serial mechanism can be constructed by the Minkowski sum of its composite sub-mechanisms provided that polytopes are defined at a common terminal point, for instance a common object. As MPs are convex the complexity of the Minkowski sum grows linearly with the number of vertices. Finally, it is important to note that the Minkowski sum is commutative $\mathcal{P}_{1}+\mathcal{P}_{2}=\mathcal{P}_{2}+\mathcal{P}_{1}$ and associative $\mathcal{P}_{1}+\left(\mathcal{P}_{2}+\mathcal{P}_{3}\right)=\left(\mathcal{P}_{1}+\mathcal{P}_{2}\right)+\mathcal{P}_{3}$ allowing a certain amount of freedom in mechanism decomposition.

\subsubsection{Closed Chains}

The analysis of closed chains can be carried out via a numerical analysis of the system's Jacobian matrix ${ }^{29}$, screw theory ${ }^{30}$, by obtaining the intersection of the velocity polytopes ${ }^{16}$ or by defining virtual kinematic chains ${ }^{31}$. To demonstrate the latter case, consider the example of two cooperating serial arms handling a rigid object. Their Cartesian-space polytopes, described by Eq. (9) when transformed to $\mathcal{H}$-representation become

$$
\mathcal{P}_{a r m 1}^{H} \triangleq \mathbf{A}_{a r m 1} \boldsymbol{\nu}_{a r m 1} \leq \mathbf{b}_{a r m 1} \quad \mathcal{P}_{a r m 2}^{H} \triangleq \mathbf{A}_{a r m 2} \boldsymbol{\nu}_{a r m 2} \leq \mathbf{b}_{a r m 2} .
$$

By simply obtaining the union of inequalities, as first shown by ${ }^{16}$, the closed-chain manipulability becomes

$$
\mathcal{P}^{H}=\left[\begin{array}{l}
\mathbf{A}_{\text {arm } 1} \\
\mathbf{A}_{\text {arm } 2}
\end{array}\right] \boldsymbol{\nu} \leq\left[\begin{array}{l}
\mathbf{b}_{\text {arm } 1} \\
\mathbf{b}_{\text {arm } 2}
\end{array}\right] .
$$

By definition $\boldsymbol{\nu}$ respects the velocity constraints imposed by both mechanisms, i.e., the intersection of the two MPs. The intersection is convex and is denoted for two MPs $\mathcal{P}_{i}, \mathcal{P}_{j}$ as $\mathcal{P}_{i \cap j}$. 


\section{Constrained Manipulability by Polytope Reduction}

In the following, the constrained manipulability polytope (CMP), which alters the manipulability polytope in response to workspace obstacles and joint position limits, is proposed.

\subsection{Obstacle constraints}

The proposed velocity reduction due to environmental constraints is guided by two principles. First, that the Cartesian velocity towards objects should be reduced in order to prevent damaging collisions. Secondly, that the velocity capacities should decrease as a link approaches an object. The kineostatic danger field ${ }^{32}$ can be used to classify how dangerous a discretized cell location in the workspace is, with respect to the robot's state. The target application is security monitoring during human robot collaboration tasks. However, in this paper, rather than creating a danger field for safety purposes, we use it as an input which limits the maximum attainable velocity toward the object. Thus, the robot's velocity is reduced until the danger-field value at the object location is below a predefined threshold.

The kineostatic danger field divides the robot's links into $l$ control points (CPs) and the workspace into $c$ cells. The danger field for the $j^{\text {th }}(j=1, \ldots, c)$ cell is calculated as

$$
\phi_{j}=\max _{i=1 \ldots l}\left(\frac{1}{\left\|\mathbf{r}_{i}-\mathbf{r}_{j}\right\|}+\frac{\left\|\mathbf{v}_{i}\right\| \cos \left(\angle\left(\mathbf{r}_{i}-\mathbf{r}_{j}, \mathbf{v}_{i}\right)\right)}{\left\|\mathbf{r}_{i}-\mathbf{r}_{j}\right\|^{2}}\right),
$$

where $\mathbf{r}_{j}$ and $\mathbf{r}_{i}$ denote the position vector of the $j^{\text {th }}$ cell and the robot's $i^{\text {th }} \mathrm{CP}$ respectively. The translational velocity of point $i$ is denoted by $\mathbf{v}_{i}$. All CPs on the robot's body can contribute to the danger value at cell $j$ rather than simply the closest point to the object. In order to create a system of inequality constraints, Eq. (20) is re-defined as

$$
\forall i \in \mathrm{CP}, \quad \phi_{j} \leq \frac{1}{\left\|\mathbf{r}_{i j}\right\|}+\frac{\left\|\mathbf{v}_{i}\right\| \cos \left(\angle\left(\mathbf{r}_{i j}, \mathbf{v}_{i}\right)\right)}{\left\|\mathbf{r}_{i j}\right\|^{2}},
$$

where $\mathbf{r}_{i j}=\mathbf{r}_{i}-\mathbf{r}_{j}$. Substituting the dot product relation

$$
\cos \left(\angle\left(\mathbf{r}_{i j}, \mathbf{v}_{i}\right)\right)=\frac{\mathbf{v}_{i}^{T} \mathbf{r}_{i j}}{\left\|\mathbf{v}_{i}\right\|\left\|\mathbf{r}_{i j}\right\|},
$$

(21) becomes

$$
\phi_{j} \leq \frac{1}{\left\|\mathbf{r}_{i j}\right\|}+\frac{\mathbf{v}_{i}^{T} \mathbf{r}_{i j}}{\left\|\mathbf{r}_{i j}\right\|^{3}},
$$

Finally, by introducing $\hat{\mathbf{r}}_{i j}$ the normalized unit vector of $\mathbf{r}_{i j}$, Eq. (23) becomes

$$
\mathbf{v}_{i}^{T} \hat{\mathbf{r}}_{i j} \leq \phi_{j}\left\|\mathbf{r}_{i j}\right\|^{2}-\left\|\mathbf{r}_{i j}\right\|,
$$

In this paper, rather than creating a danger field for safety purposes, the objective is to study the reduction in performance. Hence, the robot's velocity is reduced until 
the danger field value at the obstacle location, denoted as $o$ is below a threshold, i.e., a desired danger value. Eq. (24) is re-written as

$$
\mathbf{v}_{i}^{T} \hat{\mathbf{r}}_{i o} \leq \phi^{d}\left\|\mathbf{r}_{i o}\right\|^{2}-\left\|\mathbf{r}_{i o}\right\|,
$$

$\mathbf{r}_{o}$ is the obstacle's position vector with respect to the robot's fixed frame and $\phi^{d}$ denotes desired danger value. By introducing Eq. (1), the following expression is obtained in configuration space

$$
\hat{\mathbf{r}}_{i o}^{T} \mathbf{J}_{i} \dot{\mathbf{q}} \leq \phi^{d}\left\|\mathbf{r}_{i o}\right\|^{2}-\left\|\mathbf{r}_{i o}\right\|
$$

where $\mathbf{J}_{i} \in \mathbb{R}^{3 \times n}$ is the Jacobian matrix at point $i$. If a robot's joint does not contribute to the velocity at the $i^{\text {th }} \mathrm{CP}$, the corresponding column of $\mathbf{J}_{i}$ contains zeros. Hence, Eq. (26) constrains the maximum velocity for the $i^{\text {th }} \mathrm{CP}$ in the direction toward the obstacle. Considering the $l$ CPs leads to the following set of inequalities

$$
\left[\begin{array}{c}
\hat{\mathbf{r}}_{1 o}^{T} \mathbf{J}_{1} \\
\hat{\mathbf{r}}_{2 o}^{T} \mathbf{J}_{2} \\
\vdots \\
\hat{\mathbf{r}}_{l o}^{T} \mathbf{J}_{l}
\end{array}\right] \dot{\mathbf{q}} \leq\left[\begin{array}{c}
\phi^{d}\left\|\mathbf{r}_{1 o}\right\|^{2}-\left\|\mathbf{r}_{1 o}\right\| \\
\phi^{d}\left\|\mathbf{r}_{2 o}\right\|^{2}-\left\|\mathbf{r}_{2 o}\right\| \\
\vdots \\
\phi^{d}\left\|\mathbf{r}_{l o}\right\|^{2}-\left\|\mathbf{r}_{l o}\right\|
\end{array}\right]
$$

rewritten as

$$
\mathbf{J}_{o k} \dot{\mathbf{q}} \leq \mathbf{b}_{o}
$$

for the $k^{\text {th }}$ obstacle.

\subsection{Positional Joint Limit Constraints}

$\mathrm{In}^{8}$, the resulting manipulability value is scaled using a penalty term based on the distance to joint limits in configuration space. Alternatively, these limits can be embedded using an augmented Jacobian matrix ${ }^{3}$ or a matrix of weights ${ }^{5}$. In this paper, we introduce the effects of joint limit proximity in the polytope before transformation to the Cartesian space, thus avoiding improper penalization due to redundancy. For the $i^{t h}$ joint, the penalization term is defined as

$$
\psi_{i}^{\max }=1-\left(\frac{\max \left(\bar{q}_{i}, q_{i}\right)-\bar{q}_{i}}{q_{i}^{\text {max }}-\bar{q}_{i}}\right)^{k}, \quad \psi_{i}^{\text {min }}=1-\left(\frac{\min \left(\bar{q}_{i}, q_{i}\right)-\bar{q}_{i}}{q_{i}^{\text {min }}-\bar{q}_{i}}\right)^{k},
$$

where $\bar{q}_{i}$ is given as $\bar{q}_{i}=\frac{1}{2}\left(q_{i}^{\max }+q_{i}^{\min }\right), k$ is a positive integer, $\psi_{i}^{\max }$ varies from 1 to 0 as the $i^{\text {th }}$ joint approaches its limit. Eq. (6) is modified to consider the joint limits

$$
\left[\begin{array}{c}
\mathbf{I}_{n} \\
-\mathbf{I}_{n}
\end{array}\right] \dot{\mathbf{q}} \leq\left[\begin{array}{cc}
\boldsymbol{\Psi}^{\max } & \dot{\mathbf{q}}_{\max } \\
-\boldsymbol{\Psi}^{\min } & \dot{\mathbf{q}}_{\min }
\end{array}\right]
$$

where $\boldsymbol{\Psi}^{\max }=\operatorname{diag}\left(\psi_{1}^{\max } \ldots \psi_{n}^{\max }\right)$ and $\boldsymbol{\Psi}^{\min }=\operatorname{diag}\left(\psi_{1}^{\min } \ldots \psi_{n}^{\min }\right)$. 


\subsection{Constrained Manipulability Polytope}

By extending Eq. (28) to $m$ obstacles or danger zones in the robot's workspace and including the constraints defined by Eq. (30), the following $\mathcal{H}$-representation of the joint-space polytope, denoted as $\mathcal{Q}^{H *}$, is obtained

$$
\left[\begin{array}{c}
\mathbf{J}_{o 1} \\
\mathbf{J}_{o 2} \\
\vdots \\
\mathbf{J}_{o m} \\
\mathbf{I}_{n} \\
-\mathbf{I}_{n}
\end{array}\right] \dot{\mathbf{q}} \leq\left[\begin{array}{c}
\mathbf{b}_{o 1} \\
\mathbf{b}_{o 2} \\
\vdots \\
\mathbf{b}_{o m} \\
\mathbf{\Psi}^{\max } \dot{\mathbf{q}}_{\text {max }} \\
-\mathbf{\Psi}^{\min } \dot{\mathbf{q}}_{\text {min }}
\end{array}\right] \text {. }
$$

$\mathcal{Q}^{H *}$ can be converted to $\mathcal{V}$-representation and transformed to the task space using Eq. (1). In doing so the CMP, denoted as $\mathcal{P}^{*}$ that characterizes the constrained task-space performance while also considering joint position limits is obtained. This process results in a dependency between joint velocities as the matrix of half spaces is no longer diagonal. A performance index that considers the robot's joint limits, velocity and positional, and the constraints imposed by the environment can be obtained from measuring the volume $w_{p}^{*}$ enclosed in $\mathcal{P}^{*}$.

If it is desirable to obtain the loss of manipulability due only to the environment, $\mathcal{Q}^{*}$ must be examined. $\mathcal{Q}^{*}$ comprises $k$ n-simplexes each defined by $n$ joint velocity vertices. The volume of $\mathcal{Q}^{*}$ is given as the sum of volumes of all simplexes, i.e.,

$$
w_{q}=\sum_{s=1}^{k}\left|\frac{1}{n !} \operatorname{det}\left(\left[\dot{\mathbf{q}}_{s 1}^{v}, \dot{\mathbf{q}}_{s 2}^{v}, \ldots, \dot{\mathbf{q}}_{s n}^{v}\right]\right)\right|
$$

where $\dot{\mathbf{q}}_{s i}^{v}$ is the $i^{t h}$ vertex of the $s^{t h}$ simplex. The loss ratio is defined as

$$
\eta=\frac{w_{q}^{*}}{w_{q}}
$$

where $w_{q}^{*}$ is the volume of the deformed joint polytope. $0<\eta<1$ is dimensionless and defines the reduction in robot performance due to obstacles and proximity to joint limits. However, Eq. (33) is invalid if constraints immobilize a joint as the polytopes exist in different dimensions. Furthermore, care must be taken and appropriate scaling methods should be employed, if the mechanism has different joint types as Eq. (33) may no longer be dimensionless.

\subsection{Illustrative 2-DOF planar robot case}

In this section, the method is demonstrated for a planar 2-DOF manipulator, link lengths are given as $l_{1}=0.8, l_{2}=0.55^{\mathrm{b}}$. Additionally we compare our proposed CMP method with the extended manipulability measure ${ }^{5,14}$. Polytope manipulation

${ }^{\mathrm{b}}$ The code used to generate this example and associated figures is available here: https://github.com/philip-long/constrained_manipulation 
is performed using the Multi-Parametric Toolbox ${ }^{27}$. Figures $1 \mathrm{a}$ and $1 \mathrm{~b}$ show the manipulator in two different joint configurations. The obstacles denoted $\mathcal{O}_{1}$ and $\mathcal{O}_{2}$ are located at $\mathbf{r}_{o 1}=[0.1,0.28]$ and $\mathbf{r}_{o 2}=[0.8,0.45]$. Columns 1-4 of Fig. 1a, and Fig. $1 \mathrm{~b}$ respectively show the robot configuration followed by the MPs due to, only positional joint limits, positional joint limits with $\mathcal{O}_{1}$ and positional joint limits with $\mathcal{O}_{1-2}$. The reduction in velocity capabilities is quite small due to the joint position limits as seen in the second column for $C 1$, while for $C 2$ it is significantly higher. Indeed the combination of joint positional limits and $\mathcal{O}_{2}$ essentially immobilizes the end effector. The obstacles close to the link greatly diminishes the possible resulting velocities for the end effector. The addition of $\mathcal{O}_{2}$ has different effects on both configurations due to its relative location.

\subsubsection{Comparison with Extended Manipulability Measure}

The extended manipulability measure (EMM), first proposed in ${ }^{14}$ and later developed in ${ }^{5}$, is generated by obtaining the gradient function of constraints with respect to end-effector motions. The $m$ dimensional task space is divided into $2^{m}$ hyperoctants that represent positive and negative motions in each direction. For each motion a Jacobian matrix is constructed by penalizing the robot's unmodified Jacobian matrix. An element in a given hyperoctant's Jacobian matrix is penalized if it moves the manipulator toward a joint limit and/or obstacle. Finally the manipulability (i.e., extended inverted condition number) is obtained by computing the ratio between the minimum and maximum singular values of all augmented Jacobians. Figures $1 \mathrm{c}$ and $1 \mathrm{~d}$ show the EMM for $C 1$ and $C 2$ in the 4 translational hyperoctants, $\Gamma=\left[ \pm v_{x}, \pm v_{y}\right]$. The EMM accurately represents the restriction on motion due to joints limits in $C 2$ in the third quadrant $\Gamma=[-1,-1]$ and the presence of the $\mathcal{O}_{2}$ in $C 1$ in both second and third quadrant $\Gamma=[-1,1]$ and $\Gamma=[-1,-1]$.

Nevertheless, this example shows the advantages of the CMP. First, rather than dividing the space in hyperoctants, the constraint gradient is inherently considered by the MP's hyperplanes. Secondly, a single visualization of the extended manipulability ellipsoid requires the stitching together of each hyperoctant's ellipsoids, but this becomes problematic as the ellipsoid's major/minor axes no longer necessarily align. While it is true that the visualization does not affect the computation it can aid information transfer in a human-in-the-loop type controller ${ }^{33}$. Hence, a single visualization that encodes the directional information is an advantage of the proposed polytope method. Furthermore, since the EMM is generated by penalizing the Jacobian matrix at a single point of application, objects closer to other points on the kinematic chain have a disproportionately weak effect. This is because, in contrast to our proposed method, their effect varies with the distance to the chosen point and not the distance to the robot's body (the discretized CPs defined along the links). For instance in $C 1, \mathcal{O}_{1}$ should greatly reduce possible motion in the positive $y$ direction, whereas Fig. 1c shows that this is not the case. Likewise, for 

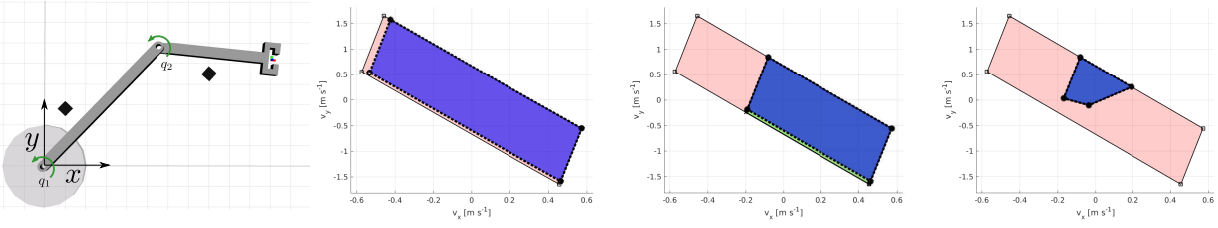

(a) CMP, $C 1: \mathbf{q}=[0.8-0.9]^{T}$
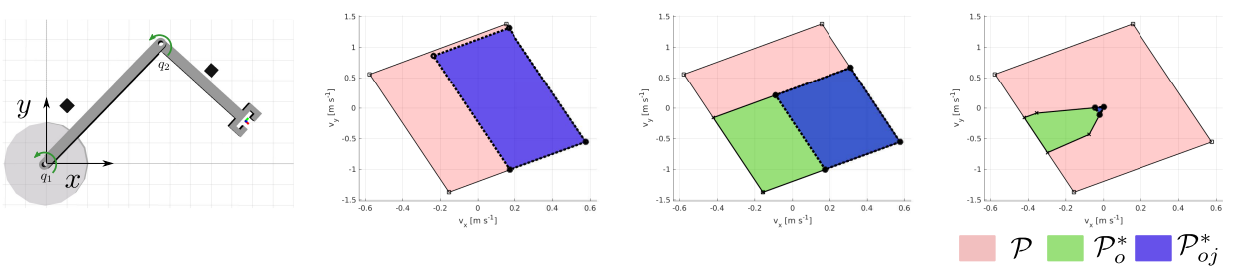

(b) CMP, $C 2: \mathbf{q}=[0.8-1.53]^{T}$.
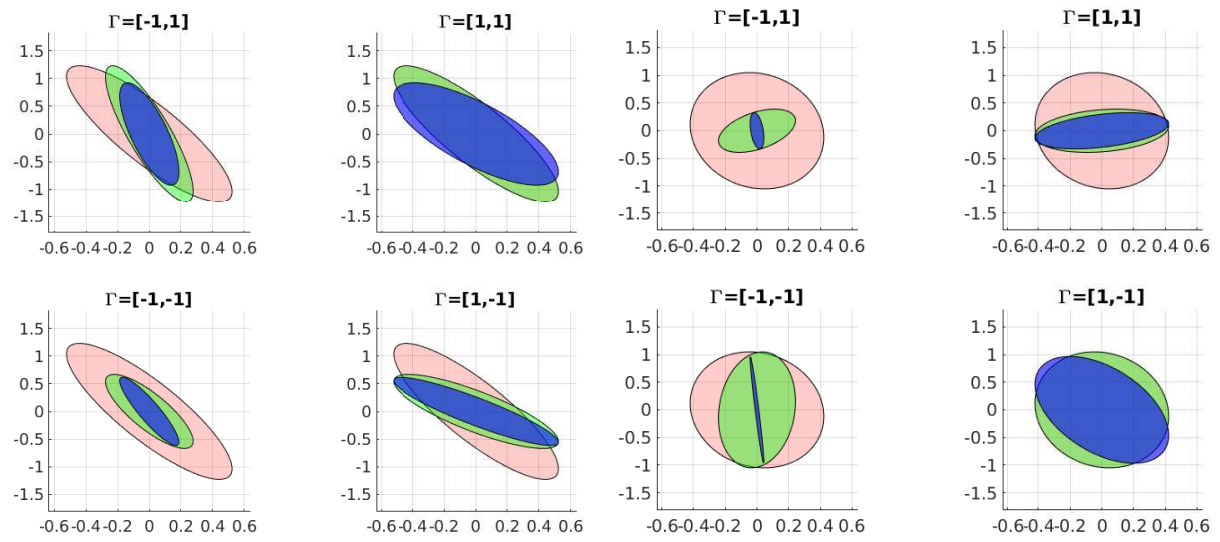

(c) $\operatorname{EMM} C 1: \mathbf{q}=[0.8-0.9]^{T}$
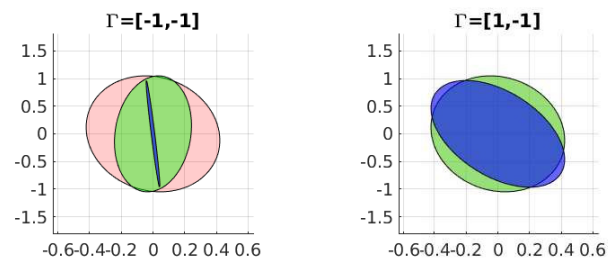

Fig. 1: The joint limits are given as $\mathbf{q}_{\max }=\left[\frac{\pi}{2}, \frac{\pi}{2}\right], \mathbf{q}_{\min }=\left[-\frac{\pi}{2},-\frac{\pi}{2}\right], \dot{\mathbf{q}}_{\min }=[-1.0$,

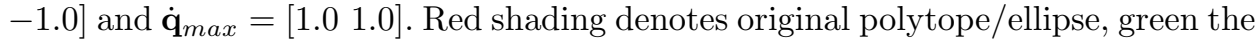
reduction due to obstacles and blue the reduction due to obstacles and joint limits. Fig. 1a and Fig. 1b, column 1 shows the robot configuration, while columns 2-4 show the effects with 0, 1, 2 obstacles, respectively. Fig. 1c and Fig 1d show the equivalent EMM in each quadrant considering both obstacles (black squares).

$C 2$, given the proximity to joint limits and the presence of objects, the manipulator is immobilized, accurately reflected in Fig. 1b, whereas Fig. 1d indicates that the motion in $\Gamma=[1,-1]$ is not impeded. On the other hand, a benefit of EMM is the existence of a closed-form solution, something which is not possible for CMP. Finally, it should be noted that the respective reduction of manipulator abilities can be altered in both methods by changing the parameter (weighting) values. 

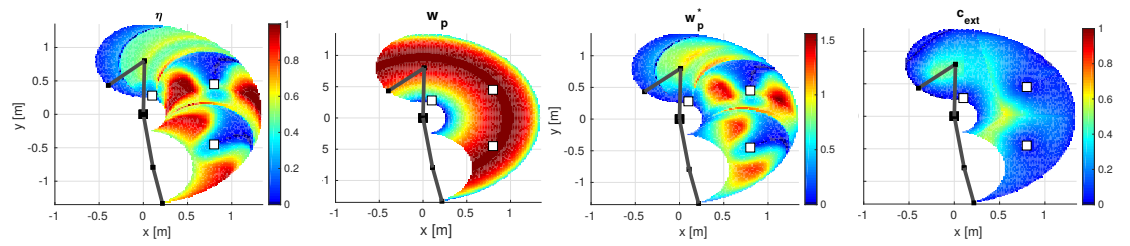

(a) Workspace Analysis $q 2 \leq 0 w_{p}$
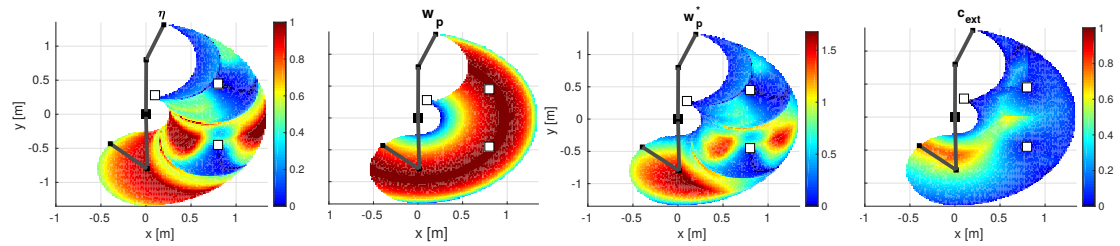

(b) Workspace Analysis $q 2 \geq 0 w_{p}$
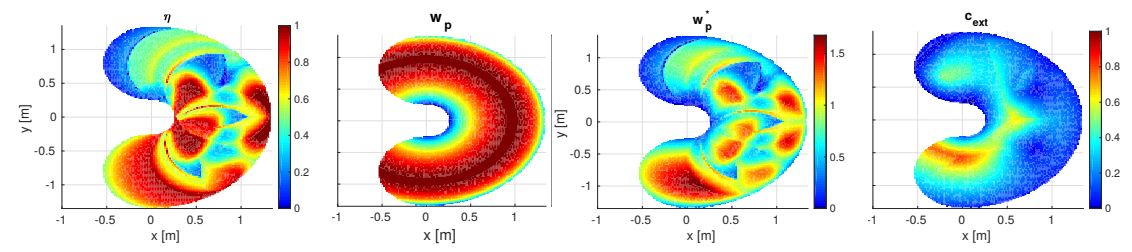

(c) Reachability Analysis

Fig. 2: Workspace analysis comparing CMP with the EMM. Red areas signify high manipulability while blue indicate the inverse. The joint position limits are $\mathbf{q}_{\max }=$ $\left[\frac{\pi}{2}, \frac{3 \pi}{2}\right] \mathbf{q}_{\text {min }}=\left[-\frac{p i}{2},-\frac{3 \pi}{2}\right]$. Rows 1-3 respectively show performance indexes for the elbow up configuration, elbow down configuration and the reachability.

\subsubsection{Workspace Analysis}

In Fig 2, the CMP is used to analyze the workspace of a robot in a cluttered environment. An inverse kinematic (IK) model obtains the possible configurations, often referred to as elbow up or elbow down at $\mathrm{mm}^{2}$ cells across the workspace. The combination of all configurations gives the system's reachability as shown in Fig. 2c. From the robot's joint configuration, obstacle location and joint limits, $\eta, w_{p}, w_{p}^{*}$ and $c_{\text {ext }}$ are calculated. Fig 2 shows how $\eta$ gives a pure representation of manipulability loss due to the obstacles, whereas $w_{p}^{*}$ also includes the effects of the manipulator's kinematic transform. It is interesting to note the dark blue curves which mark workspace boundaries, i.e, regions the manipulator cannot cross without passing through a singularity or in the reachability case cannot cross at all. Finally, it can be seen that $c_{e x t}$ increases as the end effector moves far away from obstacles, since it does not consider the effect of obstacles on the whole kinematic chain. In contrast, the variation of $w_{p}^{*}$ is more complex due to the inclusion of the constraining effects on the links. 

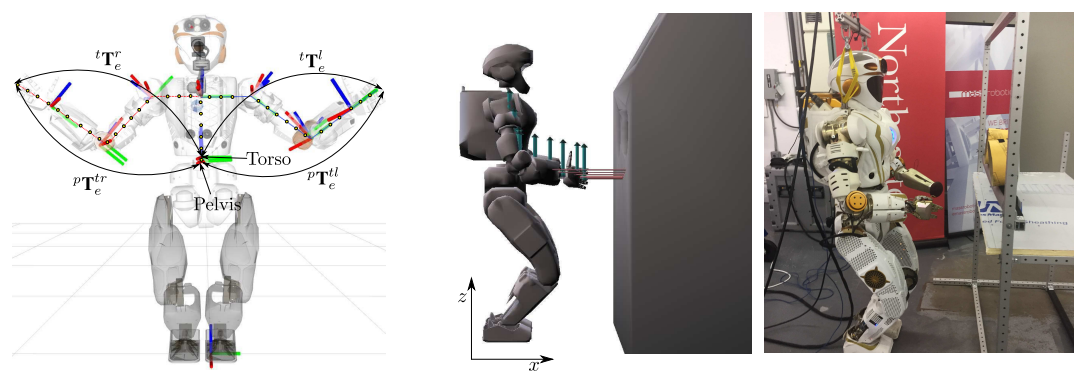

Fig. 3: NASA's Valkyrie robot. (Left) Robot model and frame location. The blue, red and yellow dotted lines denote the right-arm, left-arm and torso chains, respectively. (Right) Experimental setup, an object model (middle) is used with a ray-casting technique to obtain the minimum distance from the objects to the robot.

\section{Humanoid Robot Analysis}

In this section, the performance of a humanoid robot is evaluated in a constrained environment. In addition to the advantages detailed in Section 3.4, since the convex polytopes are geometric objects, they can be combined to represent the capacities of composite chains formed by the humanoid as outlined in Section 2.3.

Figure 3 shows the studied platform: NASA's Valkyrie robot; each arm consists of seven revolute joints whose positions are denoted by the vectors $\mathbf{q}_{r}$ and $\mathbf{q}_{l}$, while the torso comprises three revolute joints, denoted by $\mathbf{q}_{t}$. To analyze the system's manipulability, four serial chains are defined. For the two arms these chains begin at the torso frame and terminate at the index finger denoted for the right and left arms by the superscripts $r$ and $l$ respectively. To analyze the effects of the torso motion, chains begin at the pelvis frame and continue along the arms and terminate at the index fingers using the superscripts $t r$ and $t l$. The respective MPs are denoted as $\mathcal{P}_{r}, \mathcal{P}_{l}, \mathcal{P}_{t r}$ and $\mathcal{P}_{t l}$, while a CMP is denoted with an asterisk, e.g., $\mathcal{P}_{r}^{*}$. The value of $\mathbf{r}_{i o}$ from Eq. (26) is obtained by casting rays in all direction at each $\mathrm{CP}$ and checking for intersections with the environment, using a known object model.

\subsection{Right-arm insert}

In the first experiment Valkyrie must insert its right arm into a glovebox. During the action, the robot's feet are fixed and the system's MP from the torso up is measured. The glovebox is considered as an obstacle that reduces the manipulator's performance. An optimization-based motion planner ${ }^{34}$ is used to generate a collision-free trajectory for the insertion task.

Figure 4 gives the result of the right arm insertion task, showing how the CMP evolves with time. Four instants are studied corresponding to the configurations shown in Fig 4a. Rows 1-3 show $\mathcal{P}_{r}, \mathcal{P}_{t r}$ and $\mathcal{P}_{r \oplus t r}$, the polytopes corresponding to the right arm, the torso and the Minkowski sum respectively, used to obtain 

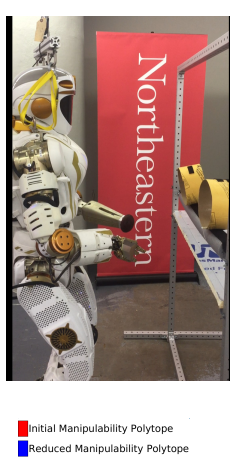
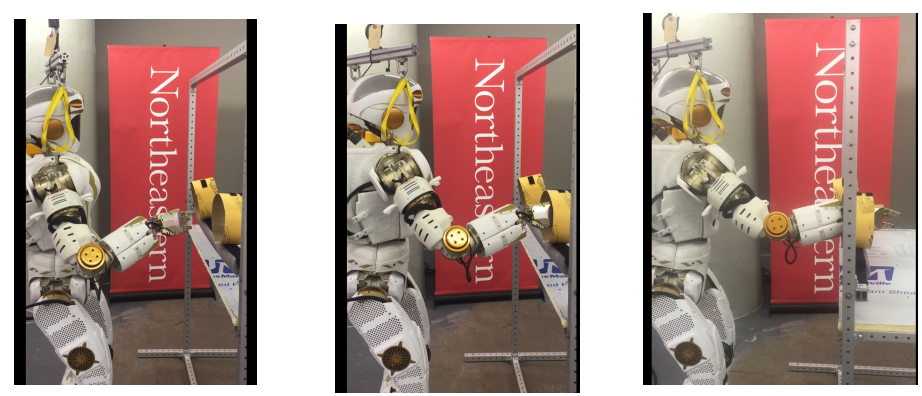

(a) Valkyrie inserting right arm into a glovebox
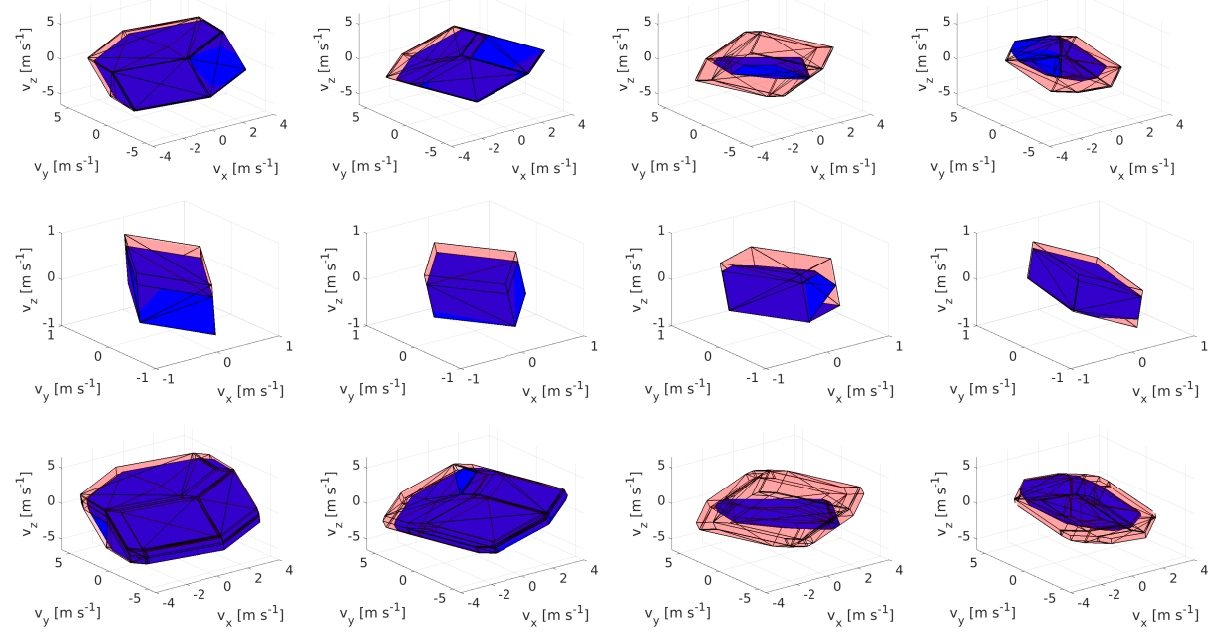

(b) Rows 1-3 respectively show the Right arm polytopes, $\mathcal{P}_{r}$ and $\mathcal{P}_{r}^{*}$; the torso polytopes $\mathcal{P}_{t}$ and $\mathcal{P}_{t}^{*}$; the Minkowksi sum $\mathcal{P}_{r \oplus t r}$ and $\mathcal{P}_{r \oplus t r}^{*}$. The columns show the evaluation at the snapshots of Fig. 4a.

Fig. 4: Single arm insertion task. MPs and CMPs are shown in red and blue respectively evaluated at the tip of the right arm. A video of the task is available https://www.youtube.com/watch?v=FzlhsLH5IPU

the composite MP/CMP of the torso chain and the right-arm serial chain. The red shows the initial MP while the blue plot shows its CMP counterpart. The initial reduction in velocity capacity is due mainly to the proximity to joint limits. As the arm passes through the port the system experiences a reduction of manipulability due to the confined space. A partial recovery is then seen as the right hand is fully inserted. $\mathcal{P}_{t r}^{*}$ undergoes the same behavior as it is also reduced due to the proximity of the glovebox to the right arm. $\mathcal{P}_{r \oplus t r}$ is the resulting CMP of the entire system. 

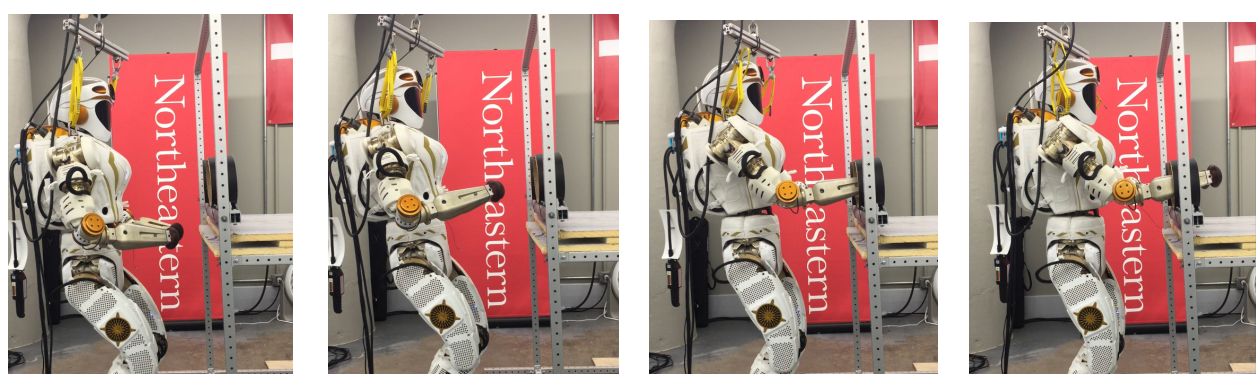

(a) Valkyrie inserting both arms into a glovebox shown at four timesteps.
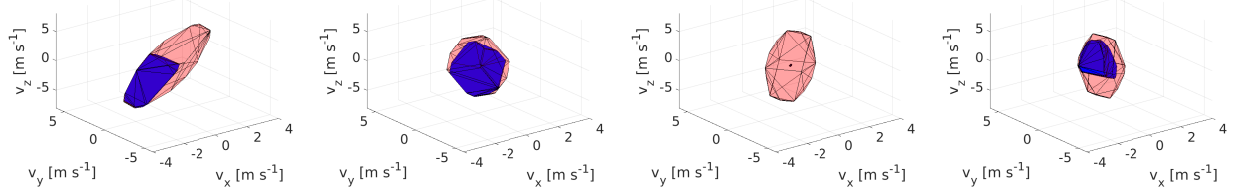

(b) Dual-arm polytopes, $\mathcal{P}_{r \cap l}$ and $\mathcal{P}_{r \cap l}^{*}$ evaluated at the right end effector. The columns 1-4 show the evaluation at the timesteps shown in Fig. 5a.

Fig. 5: Dual-arm insertion task. $\mathrm{MP}$ and CMP are shown in red and blue respectively and are evaluated at the right arm's tip. A video is available https://youtu.be/1Nouc4f_rIY

$\mathcal{P}_{r \oplus t r}^{*}$ gives the system's velocity capacities from the pelvis up, that considers joint position limits and the effects of nearby obstacles, while ensuring independence of the composite systems.

\subsection{Dual-arm insert}

In this experimental, with the same setup, Valkyrie inserts both arms into the glovebox. The goal is to demonstrate how the CMP of the arms can be combined to obtain the CMP of a closed chain system. We use the idea of a virtual object, i.e., a rigid straight link extending from the left to the right hand ${ }^{30}$. This virtual object forms part of the left arm and is discretized into CP.

Figure 5 shows the result of the dual-arm insertion task. Four time instants are studied corresponding to the configurations shown in Fig. 5a. The first row of Fig. 5b shows the CMP for the closed-chain system evaluated at the right-arm end effector. This is obtained by first calculating $\mathcal{P}_{r}$ and $\mathcal{P}_{l}$, then obtaining the intersection $\mathcal{P}_{r \cap l}$, while $\mathcal{P}_{r \cap l}^{*}$ is calculated in the same manner. In the third instant $\mathcal{P}_{r \cap l}^{*}=\emptyset$, as clearly it is impossible for the arms to enter through individual ports while holding a common object. In contrast, in the fourth instant, $\mathcal{P}_{r}^{*} \cap$ is no longer empty demonstrating the ability to co-manipulate an object within the glovebox. 

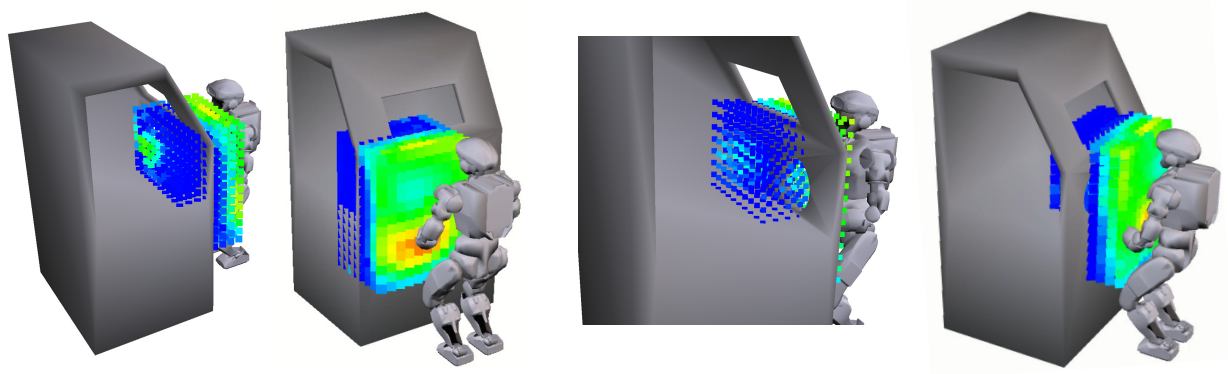

(a) The space is discretized into 3D voxels. At each voxel, the IK solution is obtained for the left arm (left images) and the right arm (right images). The corresponding volume of the CMP is calculated for each voxel, giving a good understanding of the robot's workspace.
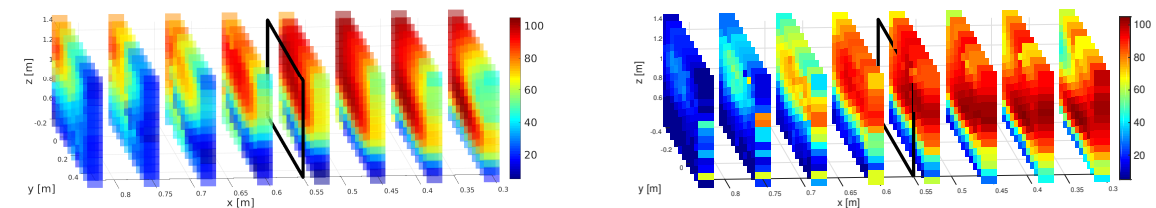

(b) The MP's volume $w_{p}$, i.e, for the left (left image) and the right (right image) end effectors. The black square shows the location of the glovebox front edge.
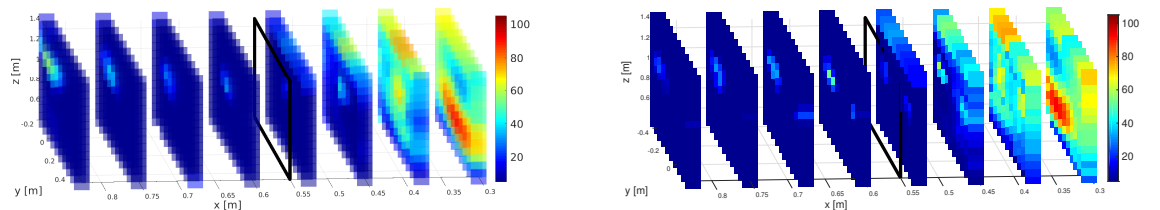

(c) The CMP's volume $w_{p}^{*}$ for the left (left image) and the right (right image) end effectors. High manipulability is possible far from the glovebox, the manipulability is extremely limited once either arm enters the glovebox.

Fig. 6: Workspace analysis using CMP, https://youtu.be/jc7X4WakdoE

\subsection{Reachability Study}

A reachability study is presented in Fig, 6 for the robot executing a glovebox task. The surrounding $3 \mathrm{D}$ space is discretized into voxels. For each voxel, an optimization procedure obtains a feasible IK solution while attempting to maximize the robot's distance to obstacles. The CMP is calculated in this configuration. The workspace discretization is shown in Fig. 6a. The voxel's color is defined by the volume of $w_{p}^{*}$. Figure $6 \mathrm{~b}$ shows the volume of $\mathcal{P}_{r}$ and $\mathcal{P}_{l}$ along the $x$-axis, i.e., along the centerline of the glovebox ports, while Fig. $6 \mathrm{c}$ shows the reduced volume for $\mathcal{P}_{r}^{*}$ and $\mathcal{P}_{l}^{*}$. The increase in manipulator capacities is clearly seen as the arm aligns with the glovebox ports. 


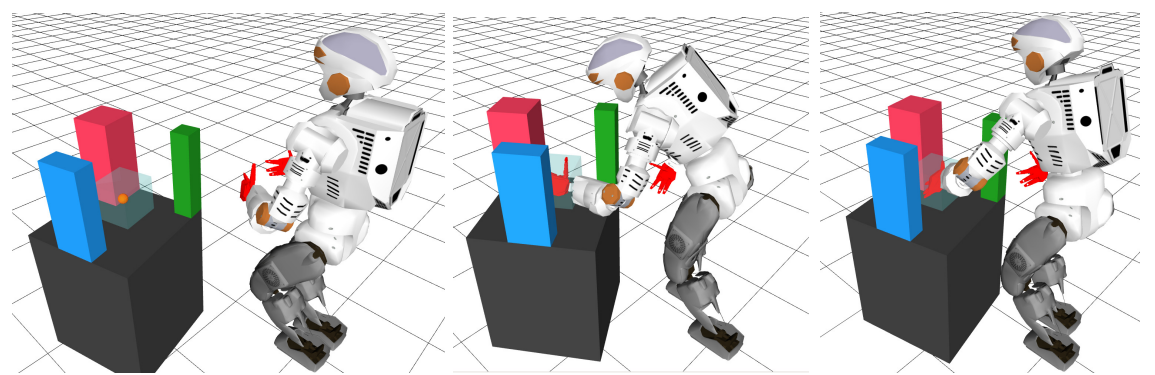

(a) For a successful run: (Left) The initial relative pose between the object (table) and the robot. The yellow ball denotes the desired position, the transparent blue region is the zone used for posture validation, the solid colored blocks and collision objects. (Middle) Posture obtained using Eq. (34). (Right) Posture obtained using MP and minimum distance as costs.

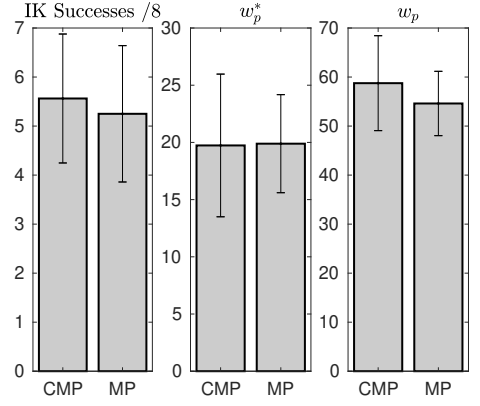

(b) A comparison of all successful runs.

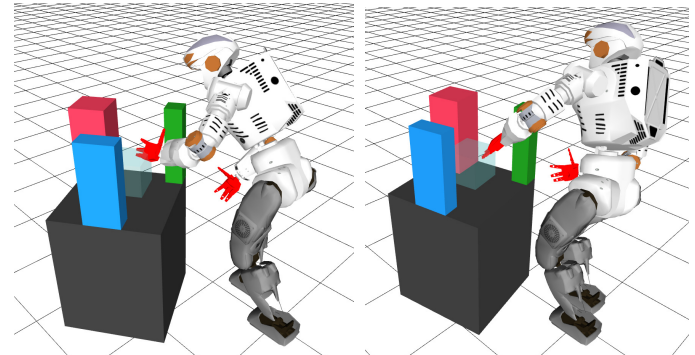

(c) Example Local IK vertex solution.

Fig. 7: Posture Optimization

\subsection{Posture Selection with Constrained Manipulability}

\subsubsection{Posture optimization}

Posture optimization is the selection of the humanoid's optimal stance for task execution. Modifying the posture mid-task is time consuming as feasible stable foot motion must be planned. This interrupts manipulation tasks, and hence it is preferable to choose a single posture from which the robot can not only execute the given tasks but adjust to task variation.

For a desired left-hand pose, $\mathbf{x}_{d}$, a whole-body IK solution, including the robot's location in the world frame, is obtained. The decision variable $\mathbf{x}_{s}$ represents the planar pose of the left foot with respect to the object frame. The robot must remain in a double support posture, but the feet may translate and rotate in the ground 
plane. The optimization problem is defined as follows:

$$
\begin{array}{cl}
\underset{\mathbf{q}, \mathbf{x}_{s}}{\operatorname{minimize}} & w_{1} e^{-b_{1} \bar{w}_{p}}+w_{2} e^{-b_{2} c_{Z M P}}+w_{3} e^{-b_{3} d_{\text {min }}}+w_{4}\left\|\mathbf{q}-\mathbf{q}_{\text {init }}\right\| \\
\text { such that } & \left\|\mathbf{x}_{d}-\mathbf{x}_{e}\left(\mathbf{q}, \mathbf{x}_{s}\right)\right\|=0, \\
& \mathbf{q}_{\text {min }} \leq \mathbf{q} \leq \mathbf{q}_{\text {max }},
\end{array}
$$

where $w_{i}$ for $i=1, \ldots, 4$ are the weights for the four cost terms defined as $w_{1}=100$, $w_{2}=w_{4}=1$ and $w_{3}=20$, and $b_{i}$ for $i=1,2,3$ are constants that determine the cost terms' curvatures defined as $b_{1}=b_{3}=40$ and $b_{2}=400$. The cost terms are designed to: (1) maximize the volume of $w_{p}^{*}$, where $\bar{w}_{p}=w_{p}^{*} / w_{p}^{\max }$ is a normalization of the CMP's volume; (2) prevent unstable postures, where $c_{Z M P}$ is the capacity margin, i.e., the signed distance from the zero-moment point ${ }^{35}(Z M P)$ to support polygon facet; (3) prevent collisions for links that are not considered in the CMP (legs, right arm etc.) by increasing the minimum distance between the robot and the environment; (4) keep the solution close to the initial configuration. The process is repeated (52 times) for different environments and random initial guesses for $\mathbf{x}_{s}$.

As a comparison the experiment is repeated, but rather than trying to maximize $w_{p}^{*}$, we maximize $w_{p}$, denoting this as MP method. Additionally, since maximizing $w_{p}^{*}$ implicitly prevents collisions, for the MP method the left arm's link frames are also included in the collision avoidance cost term.

Figure 7a shows a setup where both CMP and MP succeed. While this represents one snapshot of an array of tests, a general trend in the solutions is the parallel pose of the forearm to the table when using the CMP. We believe, this is because the MP method, penalizes the minimum distance to objects whereas the CMP simultaneously embeds the distance from all control points to the object. We believe this is also the principal reason why the MP method does not require as many random initializations (initial guess for $\mathbf{x}_{s}$ ) to find a feasible solution, typically succeeding $\sim 4 / 5$ times compared to $1 / 2$ for CMP. However, this disadvantage is mitigated without additional computational cost by using a multi-start method with parallel processing. The same weights are used for both methods, yet it is difficult to find the equivalent effects of obstacles between the classical exponential penalization term for the MP and the implicit penalization in the CMP. Thus, it is possible that both performances may be further improved by attentive tuning. Finally, the average time for the optimization using CMP is $437 s, \sigma=41 s$ compared to MP its $418 s \sigma 27 s$, information about the computation cost of the CMP can be found ${ }^{24}$.

\subsubsection{Posture validation}

The objective of this experiment is to demonstrate that $w_{p}^{*}$ encodes the environment's constraining effects and is resilient to small task position errors, i.e., should be able to find feasible poses in the neighborhood of $\mathbf{x}_{d}$. If a run is successful for CMP and MP, the posture validation procedure is executed. First, the locations of 
both feet are fixed at the final location found by the posture optimization problem. The optimizer then attempts, in series, to minimize the distance from $\mathbf{x}_{e}$ to each of the 8 vertices of the transparent cube shown in Fig. 7a, using the resulting $\mathbf{q}$ from Eq. (34) as the initial solution. The solution is constrained to collision-free and statically-stable configurations. On average as shown in Fig. 7b, the resulting posture from the CMP can find more solutions than using the MP method. Furthermore, the final solutions have a higher manipulability. Though, this is offset by the difficulty in finding the initial posture. An example IK solution is shown in Fig. 7c.

\section{Discussion \& Conclusion}

In this paper, we have extended the performance index proposed $i^{24}$, by including a detailed analysis and showing applications for a humanoid robot. The constraints are considered by introducing linear inequalities constraints in the joint velocity polytope definition. These constraints account for joint position/velocity limits and the motion restrictions due to nearby obstacles. We have shown how the CMP evolves as the humanoid robot's arm moves through a complex environment. By integrating this spatially, a workspace analysis can be obtained. Finally, we have shown how the CMP may be used as a cost in a constrained optimization problem, and how this cost can enable the optimizer to find collision-free feasible configurations.

Our approach has been demonstrated using an illustrative planar example and compared with the leading state-of-the-art method. The proposed CMP has the advantage of considering the constraints applied to the entire system and not just a single control point. Additionally, the CMP provides a natural way of visualizing the whole-body constraints in the Cartesian space for an intuitive understanding of end-effector abilities. The CMP gives exact bounds rather than the ellipsoids' estimation of capacities, this is particularly important in redundant systems, such as humanoid robots, where the manipulability ellipsoid is known to be error prone. The CMP method results in a convex geometric object which has desirable properties. For instance, we have used these properties to combine CMP's of sub-mechanisms. Thus, sub-mechanisms can be analyzed individually, while permitting a global view of the robot's capacities and ensuring constraints are satisfied. This method can be used for synthesis of composite mechanisms and reasoning considering articulated tools (by obtaining their MP). In contrast it is not trivial to combine manipulability ellipsoids and the most popular approach is to modify the Jacobian such that any additional constraints/chains are considered pre-manipulability calculation.

For Valkyrie, we limit our analysis to the upper body, however the same method can be exploited to obtain the MP that considers the whole body. For instance, given a MP of the right and left legs, denoted as $\mathcal{P}_{f l}$ and $\mathcal{P}_{f r}$, the MP at the right finger would be calculated as $\left(\mathcal{P}_{f l} \cap \mathcal{P}_{f r}\right) \oplus \mathcal{P}_{r} \oplus \mathcal{P}_{t r}$. While MP were disregarded due to computational requirements, this no longer poses such a problem. Indeed, the method's main disadvantage is the absence of a closed-form solution. 
Finally, it should be noted that translational and angular velocity polytopes should be separated as they have different units. Our examples have have focused on the translational velocities in Cartesian-space, by selecting the top three rows of Jacobian when transforming $\mathcal{Q}^{V}$. However, it can easily be extended to task directions by using the appropriate selection matrix.

Future work will focus on using CMPs to encode free space for operator assisted manipulation. In particular, we aim to generate the CMP in a receding horizon fashion to allow safe human-in-the-loop control. Finally, the MATLAB code used to generate posture optimization and planar examples is available here https://github.com/philip-long/constrained_manipulation, while the $\mathrm{C}++$ plus used for real time evaluation will be released in the future on https://github.com/RIVeR-Lab/.

\section{Acknowledgements}

This research is supported by the Department of Energy under Award Number DE-EM0004482, by the National Aeronautics and Space Administration under Grant No. NNX16AC48A issued through the Science and Technology Mission Directorate and by the National Science Foundation under Award No. 1451427.

\section{References}

1. P. Cardou, S. Bouchard, C. Gosselin, Kinematic-sensitivity indices for dimensionally nonhomogeneous jacobian matrices, IEEE Transactions on Robotics 26 (1) (2010) 166-173.

2. M. Stilman, Global manipulation planning in robot joint space with task constraints, IEEE Transactions on Robotics 26 (3) (2010) 576-584.

3. K. Abdel-Malek, W. Yu, J. Yang, Placement of robot manipulators to maximize dexterity, Int. Journal of Robotics and Automation 19 (1) (2004) 6-14.

4. T. Yoshikawa, Analysis and control of robot manipulators with redundancy, in: The first Int. symp. on Robotics research, Mit Press Cambridge, MA, 1984, pp. 735-747.

5. N. Vahrenkamp, T. Asfour, Representing the robot's workspace through constrained manipulability analysis, Autonomous Robots 38 (1) (2015) 17-30.

6. S. Kucuk, Z. Bingul, Robot workspace optimization based on a novel local and global performance indices, in: Int. Symp. on Industrial Electronics, Vol. 4, IEEE, 2005, pp. 1593-1598.

7. F. Flacco, A. De Luca, O. Khatib, Prioritized multi-task motion control of redundant robots under hard joint constraints, in: 2012 IEEE/RSJ Int.Conf. on Intelligent Robots and Systems, IEEE, 2012, pp. 3970-3977.

8. M.-J. Tsai, Workspace geometric characterization and manipulability of industrial robots, Ph.D. thesis, The Ohio State University (1986).

9. J. Lee, A study on the manipulability measures for robot manipulators, in: Int. Conf. on Intelligent Robots and Systems, Vol. 3, IEEE, 1997, pp. 1458-1465.

10. A. Bowling, O. Khatib, The dynamic capability equations: a new tool for analyzing robotic manipulator performance, IEEE Transactions on Robotics 21 (1) (2005) 115123.

11. L. Zollo, D. Accoto, F. Torchiani, D. Formica, E. Guglielmelli, Design of a planar robotic machine for neuro-rehabilitation, in: Int. Conf. on Robotics and Automation, IEEE, 2008, pp. 2031-2036. 
12. Y. Gu, C. G. Lee, B. Yao, Feasible center of mass dynamic manipulability of humanoid robots, in: Int. Conf. on Robotics and Automation, IEEE, 2015, pp. 5082-5087.

13. M. Azad, J. Babič, M. Mistry, Dynamic manipulability of the center of mass: A tool to study, analyse and measure physical ability of robots, in: IEEE Int. Conf. on Robotics and Automation, IEEE, 2017, pp. 3484-3490.

14. N. Vahrenkamp, T. Asfour, G. Metta, G. Sandini, R. Dillmann, Manipulability analysis, in: IEEE-RAS Int. Conf. on Humanoid Robots, IEEE, 2012, pp. 568-573.

15. N. Vahrenkamp, T. Asfour, R. Dillmann, Robot placement based on reachability inversion, in: 2013 Int. Conf. on Robotics and Automation, IEEE, 2013, pp. 1970-1975.

16. T. Kokkinis, B. Paden, Kinetostatic performance limits of cooperating robot manipulators using force-velocity polytopes, in: ASME Winter Annual Meeting, 1989, pp. $151-155$.

17. H. Sekiguchi, K. Ohnishi, Force capability evaluation methods for bilateral controlled manipulators, in: Int. Conf. on Mechatronics, IEEE, 2017, pp. 111-116.

18. R. Finotello, T. Grasso, G. Rossi, A. Terribile, Computation of kinetostatic performances of robot manipulators with polytopes, in: IEEE Int. Conf. on Robotics and Automation, 1998, Vol. 4, IEEE, 1998, pp. 3241-3246.

19. S. Krut, F. Pierrot, et al., Velocity performance indices for parallel mechanisms with actuation redundancy, Robotica 22 (02) (2004) 129-139.

20. J.-P. Merlet, Jacobian, manipulability, condition number, and accuracy of parallel robots, Journal of Mechanical Design 128 (1) (2006) 199-206.

21. T. Rasheed, P. Long, D. Marquez-Gamez, S. Caro, Tension distribution algorithm for planar mobile cable-driven parallel robots, in: Cable-Driven Parallel Robots, Springer, 2018, pp. 268-279.

22. T. Rasheed, P. Long, D. Marquez-Gamez, S. Caro, Available wrench set for planar mobile cable-driven parallel robots, in: 2018 IEEE Int. Conf. on Robotics and Automation, IEEE, 2018, pp. 962-967.

23. S. Caron, Q.-C. Pham, Y. Nakamura, Zmp support areas for multicontact mobility under frictional constraints, IEEE Transactions on Robotics 33 (1) (2017) 67-80.

24. P. Long, T. Padır, Evaluating robot manipulability in constrained environments by velocity polytope reduction, in: 2018 IEEE-RAS 16th Int. Conf. on Humanoid Robots, 2018.

25. I. Lee, J.-H. Oh, Humanoid posture selection for reaching motion and a cooperative balancing controller, Journal of Intelligent \& Robotic Systems 81 (3-4) (2016) 301316.

26. H. Lipkin, J. Duffy, Hybrid twist and wrench control for a robotic manipulator, Journal of Mechanisms, Transmissions, and Automation in Design 110 (2) (1988) 138144.

27. M. Herceg, M. Kvasnica, C. Jones, M. Morari, Multi-Parametric Toolbox 3.0, in: Proc. of the European Control Conf., Zürich, Switzerland, 2013, pp. 502-510.

28. K. Fukuda, A. Prodon, Double description method revisited, in: Combinatorics and computer science, Springer, 1996, pp. 91-111.

29. J. F. O'Brien, J. T. Wen, Redundant actuation for improving kinematic manipulability, in: IEEE Int. Conf. on Robotics and Automation, Vol. 2, IEEE, 1999, pp. $1520-1525$.

30. P. Long, W. Khalil, S. Caro, Kinematic and dynamic analysis of lower-mobility cooperative arms, Robotica 33 (9) (2015) 1813-1834.

31. Y. Wang, C. Smith, Y. Karayiannidis, P. Ögren, Whole body control of a dual-arm mobile robot using a virtual kinematic chain, International Journal of Humanoid Robotics 13 (01) (2016) 1550047. 
November $\quad 16, \quad 2019 \quad 14: 43 \quad$ WSPC/INSTRUCTION $\quad$ FILE

LONG ${ }^{\circ}$ IJHR $2019^{\circ}$ color

Philip Long, Taşkın Padır

32. M. Ragaglia, L. Bascetta, P. Rocco, A. M. Zanchettin, Integration of perception, control and injury knowledge for safe human-robot interaction, in: IEEE Int. Conf. on Robotics and Automation, IEEE, 2014, pp. 1196-1202.

33. P. Long, T. Keleştemur, A. Özgün Önol, T. Padır, Optimization-based human-in-theloop manipulation using joint space polytopes, in: IEEE Int. Conf. on Robotics and Automation, IEEE, 2019.

34. X. Long, M. Wonsick, V. Dimitrov, T. Padır, Task-oriented planning algorithm for humanoid robots based on a foot repositionable inverse kinematics engine, in: 2016 IEEE-RAS 16th Int. Conf. on Humanoid Robots, IEEE, 2016, pp. 1114-1120.

35. M. Vukobratović, B. Borovac, Zero-moment pointthirty five years of its life, International journal of humanoid robotics 1 (01) (2004) 157-173. 